
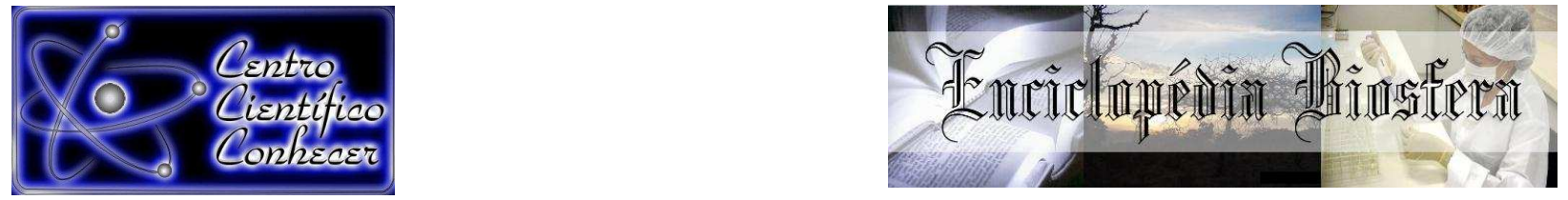

\title{
FLOTAÇÃO E MEMBRANAS ULTRAFILTRANTES COMO TRATAMENTO DE EFLUENTE ÁGUA BRANCA DE MÁQUINA DE PAPEL
}

\section{Ana Flávia Bender ${ }^{1}$; Ana Paula Bacchiega Prestes²; Bruna Luiza Managó ${ }^{3}$; Carlos Magno de Sousa Vidal ${ }^{4}$; Evandro Telegisnki ${ }^{5}$.}

${ }^{1}$ Mestranda, Pós Graduação em Ciências Florestais, Universidade Estadual do Centro-Oeste, (anabender15@gmail.com) Irati, Paraná - Brasil;

${ }^{2}$ Mestranda, Pós Graduação em Ciências Florestais, Universidade Estadual do Centro-Oeste, Irati, Paraná - Brasil;

${ }^{3}$ Doutoranda, Pós Graduação em Ciências Florestais, Universidade Estadual do Centro-Oeste, Irati, Paraná - Brasil;

${ }^{4}$ Professor Dr., Departamento de Engenharia Ambiental, Universidade Estadual do Centro-Oeste, Irati, Paraná - Brasil;

${ }^{5}$ Mestrando, Pós Graduação em Ciências Florestais, Universidade Estadual do Centro-Oeste, Irati, Paraná - Brasil;

Recebido em: 03/10/2016 - Aprovado em: 21/11/2016 - Publicado em: 05/12/2016 DOI: 10.18677/EnciBio_2016B_051

\begin{abstract}
RESUMO
O setor de papel e celulose contribui fortemente na economia do país. O alto consumo de água que essas empresas geram implica diretamente em altas vazões de efluentes a serem tratados. Devido à recalcitrância e o interesse no reuso desses efluentes torna-se interessante processos de tratamento mais sofisticados. $O$ objetivo do estudo foi avaliar a eficiência dos sistemas de membranas filtrantes, bem como comparar a utilização destas no tratamento da água branca advinda da máquina de papel na forma bruta com um sistema de ultrafiltração que receba o efluente pré-tratado através da flotação por ar dissolvido. A comparação entre esses sistemas de tratamento foi realizada através dos seguintes parâmetros: turbidez, cor aparente, demanda química de oxigênio e fluxo do permeado. Os experimentos foram realizados seguindo quatro etapas, sendo estas: caracterização do efluente, pré-tratamento da água branca por flotação, tratamento por ultrafiltração do efluente clarificado proveniente do pré-tratamento por flotação e tratamento da água branca por ultrafiltração. As análises foram realizadas conforme métodos estabelecidos no Standard Methods for the Examination of Water and Wastewater. Nos tratamentos de ultrafiltração variou-se o tempo de coleta do permeado em 20, 40, 60, 80, 100 e 120 min. Os resultados obtidos apontaram que ambos os tratamentos alcançaram resultados satisfatórios, porém a utilização de um pré-tratamento, principalmente no que diz respeito ao fluxo do permeado, é indicado a fim de garantir o bom desempenho das membranas. Também indica-se a adição de coagulante na etapa de floculação com finalidade de remover teores maiores de sólidos dissolvidos.
\end{abstract}

PALAVRAS-CHAVE: Água branca. Análises físico-químicas. Indústria papeleira. 


\section{FLOTATION AND ULTRAFILTRATION MEMBRANES AS TREATMENT OF WHITE WATER WASTEWATER FROM PAPER MACHINE ABSTRACT}

The pulp and paper industry strongly contributes to the economy. The high water consumption that these companies generate directly implies high flow of effluents to be treated. Because of recalcitrance and interest in the reuse of this effluent becomes interesting more sophisticated treatment processes. The aim of the study was to evaluate the efficiency of the ultrafiltration system, and to compare the use of these in the treatment of white water from a paper machine in raw form with an ultrafiltration system that receives the pre-treated effluent through the air flotation dissolved. The comparison between these treatment systems was performed using the following parameters: turbidity, apparent color, chemical oxygen demand and permeate flow. The experiments were performed following four steps, which are: characterization of the effluent, pre-treatment of white water by flotation treatment by ultrafiltration of the clarified effluent from the pretreatment by dissolved air flotation and treatment of raw white water by ultrafiltration. Analyses were performed according to established methods in Standard Methods for the Examination of Water and Wastewater. In the ultrafiltration treatment time collection of the permeate was varied in 20,40,60, 80, 100 and $120 \mathrm{~min}$. The results showed that both treatments achieved satisfactory results, but the use of a pre-treatment, particularly as regards the permeate flow is indicated in order to ensure the good performance of the membranes. It also indicates the addition of coagulant in the flocculation step in order to remove higher levels of dissolved solids.

KEYWORDS: White Water. Physicochemical analysis. Paper industry.

\section{INTRODUÇÃO}

O Brasil encontra-se dentre os maiores produtores mundiais de papel e celulose, ocupando a $4^{\mathrm{a}}$ colocação no ranking quanto à produção de celulose e $9^{\mathrm{a}}$ na produção de papel (IBÁ, 2015). Dessa forma corresponde a um setor de enorme importância, contribuindo significativamente com a economia do país.

Além do destaque econômico, as indústrias desse setor são conhecidas pela tradicional contribuição para os impactos ambientais devido ao grande consumo de energia e produtos químicos, bem como pela geração de efluentes com elevados teores de sólidos em suspensão, matéria orgânica e toxicidade. (PACHECO \& SOARES, 2011). Especificamente a parte úmida da produção, chamada de água branca, é muito problemática, devido à coloração produzida por sólidos em suspensão e outros componentes do papel perdidos no efluente, como: cola, amido, carbonato de cálcio e corantes (SOUSA et al., 2011).

A indústria de papel e celulose comumente utiliza-se de processos biológicos para tratar os efluentes, principalmente lodos ativados e lagoas aeróbias, as quais apresentam elevada eficiência na remoção de matéria orgânica biodegradável, mas que deixam a desejar quanto à remoção de matéria orgânica recalcitrante e a outros poluentes presentes nesse tipo de efluente (JUDD, 2016). Devido a estas limitações outras tecnologias, capazes da aumentar a eficiência desses processos vem sendo implementadas nas fábricas. Dentre estas tecnologias pode-se citar o processo de flotação por ar dissolvido e a ultrafiltração (METCALF \& EDDY, 2003).

Segundo QUARTAROLI et al., (2014) a flotação pode ser descrita como um sistema no qual é possível separar partículas sólidas ou líquidas de uma fase líquida a partir da introdução de pequenas bolhas de gás. Essas bolhas aderem-se a superfície das partículas, aumentando o empuxo, favorecendo assim o movimento 
ascensional em direção à superfície da fase líquida, onde poderão ser coletadas através de raspadores superficiais.

Outra tecnologia que tem sido uma ótima opção de tratamento de efluente de industrial de maneira geral são as membranas filtrantes. Segundo JUDD \& JEFFERSON (2003) trata-se de uma técnica de filtração em que é aplicada uma força exterior sobre uma membrana semipermeável, capaz de reter substâncias de diâmetro maior que os poros das membranas, gerando um efluente concentrado com os contaminantes presentes no efluente, e um permeado de alta qualidade.

Ainda segundo JUDD \& JEFFERSON (2003), a qualidade do permeado depende das características do efluente, e principalmente, do tipo de membrana escolhida para filtração, sendo que a seletividade da membrana está ligada às dimensões do poro, e que os poros com menores diâmetros apresentam maior capacidade de retenção dos poluentes presentes no efluente.

Pensando nisso, para manter o desempenho da membrana é importante fazer limpezas frequentes, não permitindo que os poros da membrana sejam obstruídos por materiais indesejáveis. Segundo GUO et al., (2012) essa obstrução, caracterizase pela formação de uma camada de polarização, formação de camada de gel e, também pela colmatação por adsorção ou penetração, causando o bloqueamento dos poros.

Com legislações cada vez mais restritivas quanto aos padrões legais de lançamento de efluente, bem como, com o interesse apresentado pelas indústrias quanto ao reuso no próprio processo produtivo, é de fundamental importância que se busque aperfeiçoar, cada vez mais, a qualidade final do efluente. Isto pode ser feito, por exemplo, por meio de modificações nos sistemas de tratamento (NEOH et al., 2016).

Neste contexto, o estudo objetivou avaliar a eficiência dos sistemas de membranas filtrantes, bem como comparar a utilização de membranas ultrafiltrantes no tratamento da água branca advinda da máquina de papel na forma bruta com um sistema de ultrafiltração que receba o efluente pré-tratado com flotação por ar dissolvido.

\section{Efluente água branca}

\section{MATERIAL E MÉTODOS}

O efluente denominado água branca foi coletado na canaleta de saída, da parte úmida, da máquina de papel, proveniente de uma indústria de papel tipo offset, da região de Guarapuava-PR.

\section{Experimentos}

Os experimentos foram realizados seguindo 4 etapas, todos em triplicata:

\section{Etapa 1: Caracterização do efluente água branca}

As amostras do efluente água branca bruto foram analisadas para os seguintes parâmetros: turbidez, cor verdadeira, cor aparente, demanda química de oxigênio (DQO), sólidos totais (ST) e sólido suspensos totais (SST). Conforme métodos estabelecidos no Standard Methods for the Examination of Water and Wastewater (APHA, 2005).

Etapa 2: Tratamento da água branca por flotação

O efluente, primeiramente, foi aquecido a aproximadamente $35{ }^{\circ} \mathrm{C}$, a fim de simular a situação real do mesmo na indústria, e submetido ao tratamento físico de 
flotação em equipamento flotateste, com operação em batelada, de acordo com a metodologia proposta por DI BERNARDO et al., (2002). Variaram-se as seguintes porcentagens de recirculação: 0, 10, 15 e $20 \%$, para verificar o desempenho da flotação por ar dissolvido.

Os parâmetros fixados para a realização dos ensaios de flotação estão listados abaixo na Tabela 1. Esses parâmetros foram adotados segundo valores encontrados na literatura (QUARTAROLI et al., 2014; MANAGÓ et al., 2016).

TABELA 1. Parâmetros adotados para os ensaios de flotação por ar dissolvido para o tratamento de efluente água branca.

\begin{tabular}{cc}
\hline Parâmetro & Configuração \\
\hline Tempo de coleta & $1 \mathrm{~min}$ e $20 \mathrm{seg}$ \\
Velocidade ascensional & $9 \mathrm{~cm} / \mathrm{min}$ \\
Tempo de saturação & $15 \mathrm{~min}$ \\
Pressão de saturação & $5 \mathrm{bar}$ \\
\hline
\end{tabular}

Os parâmetros analisados na fase de otimização da taxa de recirculação foram: turbidez e cor aparente. De acordo com os melhores resultados encontrados, escolheu-se a porcentagem de recirculação mais adequada a ser aplicada como pré-tratamento. O efluente clarificado foi coletado na parte inferior do jarro de flotação para posterior tratamento no equipamento de UF.

Etapa 3: Tratamento da água branca por ultrafiltração (UF)

Os ensaios na unidade de bancada de membranas UF foram realizados em escala laboratorial com equipamento adquirido da PAM Membranas Seletivas Ltda. A membrana utilizada foi de geometria cilíndrica, do tipo fibra oca, fixada na carcaça de policloreto de vinila (PVC). Durante os experimentos, o equipamento de bancada de UF foi operado com recirculação total de permeado para o reservatório de alimentação, salvo quando necessária à coleta de amostras para análises físicoquímicas. Na Tabela 2 são apresentadas as características do módulo de ultrafiltração testados na pesquisa.

TABELA 2. Propriedades do módulo de UF utilizado nos testes.

\begin{tabular}{cc}
\hline Propriedades & Ultrafiltração \\
\hline Material da membrana & Poli (éter sulfona) \\
Comprimento da fibra $(\mathrm{cm})$ & 26 \\
Diâmetro da fibra $(\mathrm{mm})$ & 25 \\
Tamanho médio dos poros & $50 \mathrm{kda}$ \\
Empacotamento $\left(\mathrm{m}^{2} / \mathrm{m}^{3}\right)$ & 1000 \\
Área de filtração $\left(\mathrm{m}^{2}\right)$ & 0,090 \\
\hline
\end{tabular}

Os seguintes tempos de coleta foram variados durante os tratamentos: 20,40 , 60, 80, 100 e 120 min. Os parâmetros analisados nas amostras coletadas nos tempos de tratamento variados foram: turbidez, cor aparente, DQO e fluxo do permeado. Os parâmetros fixados para a realização dos ensaios de ultrafiltração estão listados abaixo na Tabela 3. Esses parâmetros foram adotados segundo valores encontrados na literatura (AMARAL et al., 2013). 
TABELA 3. Parâmetros adotados para os ensaios de ultrafiltração para o tratamento de efluente água branca.

\begin{tabular}{cc}
\hline Parâmetro & Configuração \\
\hline Tempo de operação & 2 horas \\
Pressão & 0,25 bar \\
Vazão & $1,6 \mathrm{~L} / \mathrm{min}$ \\
Retrolavagem & $45 \mathrm{seg}($ a cada $10 \mathrm{~min})$ \\
\hline
\end{tabular}

Etapa 4: Tratamento por ultrafiltração do efluente clarificado proveniente do prétratamento por flotação da Etapa 2

O efluente coletado na Etapa 2 foi acondicionado no tanque de alimentação do equipamento e passou pelo tratamento de UF, seguindo as mesmas condições e parâmetros utilizados na Etapa 3.

Métodos estatísticos utilizados na pesquisa

Foi utilizado o software livre e gratuito Assistat 7.7 beta, no qual foi realizado o teste de homogeneidade de Bartlett e teste de normalidade de Shapiro-Wilk à $5 \%$ de significância. Após a análise de variância foram realizados testes de comparações múltiplas de médias para cada parâmetro, pelo teste de Tukey, com um nível de significância de 5\%.

\section{RESULTADOS E DISCUSSÃO}

\section{Etapa 1: Caracterização do efluente água branca bruto}

A Tabela 4 demonstra os resultados da primeira etapa, a qual corresponde à caracterização do efluente antes de submetê-lo aos tratamentos. Nesta, pode-se notar valores elevados de sólidos, DQO e cor.

TABELA 4. Características da amostra do efluente bruto

\begin{tabular}{cc}
\hline Parâmetro & Configuração \\
\hline pH & 6,8 \\
Temperatura & $35\left({ }^{\circ} \mathrm{C}\right)$ \\
Turbidez & $72,1(\mathrm{uT})$ \\
Cor Aparente & $475,3(\mathrm{uC})$ \\
Cor Verdadeira & $45,6(\mathrm{uC})$ \\
DQO & $363,4\left(\mathrm{mg} \cdot \mathrm{L}^{-1}\right)$ \\
Sólidos Totais & $590\left(\mathrm{mg} \cdot \mathrm{L}^{-1}\right)$ \\
Sólidos Suspensos Totais & $298\left(\mathrm{mg} \cdot \mathrm{L}^{-1}\right)$ \\
\hline
\end{tabular}

\section{Etapa 2: Tratamento da água branca por flotação}

A partir dos resultados obtidos no ensaio de flotação pode-se fazer a escolha da porcentagem de recirculação que melhor removeu os parâmetros turbidez e cor aparente. Essa determinação se deu a partir do teste ANOVA a 5\% de significância e do teste de comparação de médias Tukey. Na tabela 5 encontram-se os resultados do teste de Tukey para os parâmetros turbidez remanescente e cor aparente nas diferentes porcentagens de recirculação. 
TABELA 5. Médias obtidas após o tratamento de flotação para os parâmetros: turbidez, cor aparente.

\begin{tabular}{ccccc}
\hline \multicolumn{5}{c}{ Recirculação (\%) } \\
Parâmetros & 0 & 10 & 15 & 20 \\
\hline Turbidez (uT) & $74,26 \mathrm{a}^{*}$ & $22,06 \mathrm{~b}$ & $22,9 \mathrm{~b}$ & $21,2 \mathrm{~b}$ \\
Cor Ap. (uC) & $360,33 \mathrm{a}$ & $184 \mathrm{~b}$ & $204,66 \mathrm{~b}$ & $192,66 \mathrm{~b}$ \\
\hline
\end{tabular}

* Médias seguidas da mesma letra não diferem estatisticamente entre si, pelo teste de Tukey a $5 \%$ de probabilidade.

De acordo com a tabela anterior, pode-se verificar que ambos os parâmetros demonstraram diferença estatística significativa entre $0 \%$ de recirculação e as médias obtidas para as demais porcentagens. Sendo assim, as menores médias para os parâmetros analisados pertencem as recirculações de 10, 15 e 20\%, as quais não diferem entre si. Dessa forma, pensando-se num menor volume útil de reator, opta-se pela menor porcentagem de recirculação (10\%).

\section{Etapa 3: Tratamento da água branca por ultrafiltração}

Os testes estatísticos realizados para esta etapa de tratamento podem ser observados na Tabela 6 . Pode-se notar que os valores médios encontrados para 0 parâmetro DQO diferiram estatisticamente conforme os tempos de coleta, indicando que o tratamento conseguiu remover matéria orgânica ao longo do tempo de filtração.

Para os parâmetros turbidez, cor aparente e fluxo os resultados são estatisticamente iguais para todos os tempos de coleta. No entanto, ao observar os valores obtidos para o fluxo, percebe-se que conforme o tempo de coleta aumenta 0 valor do fluxo decai, indicando uma possível colmatação da membrana.

TABELA 6. Médias obtidas nos respectivos tempos de coletas para o tratamento por ultrafiltração.

\begin{tabular}{|c|c|c|c|c|c|c|}
\hline \multicolumn{7}{|c|}{ Tempo de coleta do permeado (min) } \\
\hline Parâmetros & 20 & 40 & 60 & 80 & 100 & 120 \\
\hline Turbidez (uT) & $0.93 a^{*}$ & $0.95 \mathrm{a}$ & $1.40 \mathrm{a}$ & $0.87 \mathrm{a}$ & $1.013 \mathrm{a}$ & $2.086 \mathrm{a}$ \\
\hline Cor Ap. (uC) & $15 \mathrm{a}$ & $13 a$ & $14.33 \mathrm{a}$ & $10.33 \mathrm{a}$ & $11.66 \mathrm{a}$ & $20.33 \mathrm{a}$ \\
\hline DQO $\left(\mathrm{mg} \cdot \mathrm{L}^{-1}\right)$ & $96.42 \mathrm{a}$ & $89 a b c$ & $90.42 \mathrm{ab}$ & $74.05 \mathrm{bc}$ & $74.15 \mathrm{bc}$ & $70.66 \mathrm{c}$ \\
\hline Fluxo & $10.12 \mathrm{a}$ & $8.51 \mathrm{a}$ & $7.32 \mathrm{a}$ & $6.46 \mathrm{a}$ & $6.12 \mathrm{a}$ & $5.63 \mathrm{a}$ \\
\hline
\end{tabular}

* Médias seguidas da mesma letra não diferem estatisticamente entre si, pelo teste de Tukey a $5 \%$ de probabilidade.

Ao analisar o tratamento de efluente da máquina do papel e da máquina de secagem da indústria de papel e celulose por ultrafiltração, OLIVEIRA (2003) encontrou valores semelhantes para os parâmetros turbidez e DQO, com remoções de $95 \%$ e $37 \%$ respectivamente. A redução da turbidez foi de 61,2 para 2,9 e DQO de 284 para 179. Este autor também verificou a queda de fluxo ao longo do tratamento por ultrafiltração, indicando colmatação progressiva das membranas. Outro trabalho utilizando membranas filtrantes foi o desenvolvido por REYES (2013) para tratar efluentes da planta de branqueamento, seus resultados indicaram a remoção de $64 \%$ da DQO e $87 \%$ da cor. 


\section{Etapa 4: Tratamento por ultrafiltração do efluente clarificado proveniente do pré-tratamento por flotação da Etapa 2.}

De acordo com o teste de médias Tukey foi possível identificar entre os resultados obtidos na Etapa 4, que não houve diferença estatística para todos os tempos de coleta nos parâmetros: turbidez, DQO e fluxo (Tabela 7). Já para a cor aparente houve uma diferença estatística, sendo que os tempos de 20 e 40 minutos tiveram resultados mais satisfatórios.

TABELA 7. Médias obtidas nos respectivos tempos de coletas para o pré-tratamento por flotação seguido de ultrafiltração.

\begin{tabular}{ccccccc}
\hline \multicolumn{7}{c}{ Tempo de coleta (min) } \\
Parâmetros & 20 & 40 & 60 & 80 & 100 & 120 \\
\hline Turbidez (uT) & $0.46 \mathrm{a}{ }^{*}$ & $0.56 \mathrm{a}$ & $0.54 \mathrm{a}$ & $0.86 \mathrm{a}$ & $0.54 \mathrm{a}$ & $0.54 \mathrm{a}$ \\
Cor Ap. (uC) & $2 \mathrm{~b}$ & $2.66 \mathrm{~b}$ & $3.66 \mathrm{ab}$ & $3.66 \mathrm{ab}$ & $6 \mathrm{a}$ & $5 \mathrm{ab}$ \\
DQO (mg. (m) & $80.41 \mathrm{a}$ & $79.54 \mathrm{a}$ & $74.22 \mathrm{a}$ & $75.66 \mathrm{a}$ & $73.51 \mathrm{a}$ & $80.9 \mathrm{a}$ \\
Fluxo & $15.77 \mathrm{a}$ & $16.36 \mathrm{a}$ & $16.16 \mathrm{a}$ & $16.78 \mathrm{a}$ & $16.50 \mathrm{a}$ & $16.69 \mathrm{a}$ \\
\hline
\end{tabular}

* Médias seguidas da mesma letra não diferem estatisticamente entre si, pelo teste de Tukey a $5 \%$ de probabilidade.

A fim de comparar os dois tratamentos estudados (UF e Flotação+UF) gerouse os gráficos da Figura 1. Pode-se observar que a ultrafiltração obteve melhores resultados quando à esta aplicado um pré-tratamento. Porém quando estes resultados são comparados com o efluente bruto (Tabela 4) nota-se que para ambos tratamentos os resultados foram excelentes no que diz respeito à remoção de turbidez (a) e cor aparente (b). Para o parâmetro DQO (c) as remoções foram menores, isto pode estar relacionado com os sólidos solúveis presentes no efluente, os quais apresentam parcelas de amido, cola, carbonato de cálcio e corantes.
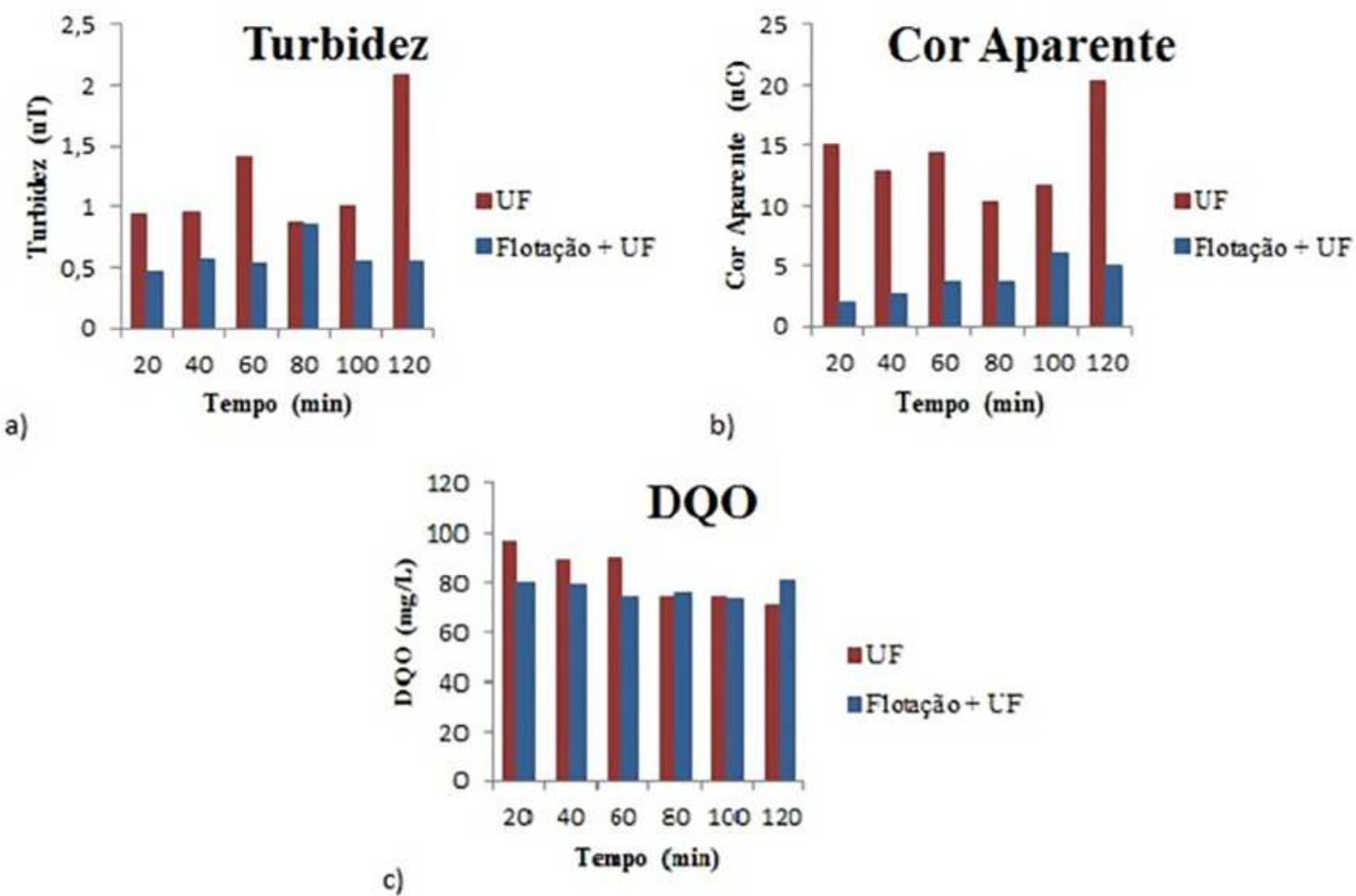

FIGURA 1. Comparação entre os tratamentos da Etapa 3 e 4, para os parâmetros turbidez, Cor e DQO.

Fonte: Os autores (2016). 
ALMEIDA (2002) ao estudar o tratamento alcalino do branqueamento da polpa obteve $95 \%$ da remoção de fibras através da flotação, e atestou que ainda havia presença de partículas grosseiras no efluente, as quais, possivelmente, causariam danos quanto a colmatação das membranas utilizadas nos ensaios posteriores. Segundo QU et al. (2012), a colmatação da membrana é um dos principais fenômenos responsáveis por diminuir o fluxo do permeado.

Nesse sentido, QUARTAROLI (2012) assegurou que, quando utiliza-se o sistema de flotação por ar dissolvido, ocorre a remoção de partículas coloidais e dissolvidas reduzindo a colmatação dos poros da membrana, aumentando o fluxo de permeado. Isso também pode ser observado na figura abaixo (Figura 2).

\section{Evolução dos Fluxos de Permeado nos Ensaios de Ultrafiltração}

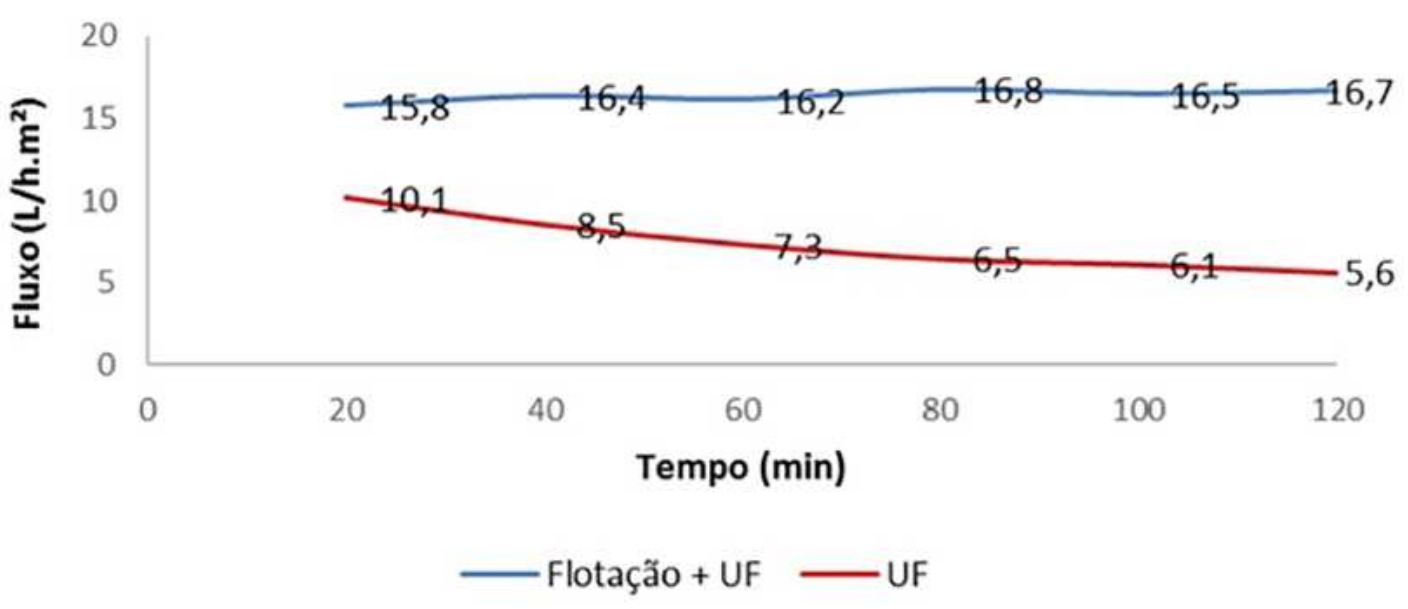

FIGURA 2. Evolução dos fluxos de permeado nos ensaios de ultrafiltração.

Fonte: Os autores (2016).

Pode-se perceber que o sistema que recebe um pré-tratamento apresenta valores para fluxo mais estáveis, sem que ocorra o decaimento do mesmo. Enquanto que para o tratamento por ultrafiltração do efluente bruto o fluxo decai com - passar do tempo de filtração, indicando colmatação da membrana e consequentemente perdas no que diz respeito a volumes tratados por tempo além de aumentos no consumo de energia.

NUORTILA-JOKINEN et al., (2004) afirmam que os processos de membrana podem ser melhorados por várias técnicas de pré-tratamento para produzir fluxos de permeado mais elevados e para eliminar a colmatação da membrana. Tais métodos de pré-tratamento são, por exemplo, floculação, flotação, ozonização e tratamento biológico.

\section{CONCLUSÃO}

A partir dos resultados obtidos nos experimentos podemos concluir que:

- Ambos tratamentos alcançaram resultados satisfatórios;

- A flotação por ar dissolvido é recomendada como pré-tratamento da UF, pois ao analisar a tendência do fluxo do permeado, o pré-tratamento do efluente colabora com a redução do mecanismo de colmatação de membrana, tendo vários benefícios, como o aumento do tempo útil das 
membranas, menor gastos com limpezas químicas e melhora na taxa de fluxo.

- Devido aos resultados obtidos para a DQO, indica-se a adição de coagulante na etapa de floculação, com o propósito de que uma maior parcela de sólidos solúveis formem flocos e sejam removidos nesta etapa, aumentando a eficiência final.

\section{REFERÊNCIAS}

ALMEIDA, K. M. Tratamento do efluente alcalino do branqueamento de polpa e celulose pelo processo de separação por membranas. 2002. 139 p. Dissertação (Mestrado em Engenharia Química) - Universidade Federal do Rio Grande do Sul, Porto Alegre,RS.

AMARAL, M.C.S.; ANDRADE, L.H.; LANGE, L.C.; BORGES, C.P. Avaliação do emprego de microfiltração para remoção de fibras do efluente de branqueamento de polpa celulósica. Engenharia Sanitária e Ambiental, v.18, n.1, p.65-74. 2013. Disponível em: <http://www.scielo.br/scielo.php?script=sci_arttext\&pid=S141341522013000100008\&Ing=en\&nrm=iso> Acesso em: 26 ago. 2016. doi: $10.1590 / S 1413-41522013000100008$.

APHA - AMERICAN PUBLIC HEALTH ASSOCIATION. Standard Methods For The Examination Of Water And Wastewater, 21 ed., Washington, 2005.

DI BERNARDO, L.; DI BERNARDO, A.; CENTURIONE FILHO, P. L. Ensaios de Tratabilidade de Água E Dos Resíduos Gerados Em Estações de Tratamento De Água. São Carlos: Rima, 233 p. 2002.

GUO, W.; NGO, H-H.; LI, J. A mini-review on membrane fouling. Bioresource Technology, 122, p.27-34. 2012. Disponível em: <http://dx.doi.org/10.1016/j.biortech.2012.04.089> Acesso em: 26 ago. 2016. doi: 10.1016/j.biortech.2012.04.089.

INDÚSTRIA BRASILEIRA DE ÁRVORES - IBA. Relatório anual 2016. Disponível em: <http://iba.org/images/shared/Biblioteca/IBA_RelatorioAnual2016_.pdf> Acesso em: 22 ago. 2016.

JUDD, S.; JEFFERSON, B. Membranes for industrial wastewater recovery and reuse. New York: Elsevier Science, p.291, 2003.

JUDD, S. The status of industrial and municipal effluent treatment with membrane bioreactor technology. Chemical Engineering Journal, v.305, p.37-45. 2016. Disponível em: < http://dx.doi.org/10.1016/j.cej.2015.08.141> Acesso em: 14 nov. 2016. doi: 10.1016/j.cej.2015.08.141

MANAGÓ, B.L; SOUSA, C.M.; VIDAL, SOUZA, J.B. Dissolved air flotation for recovering fibers from clear water of a paper machine. Aust. J. Basic \& Appl. Sci., 10(12): 354-361, 2016. Disponível em: <http://ajbasweb.com/old/ajbas/2016/July/354-361.pdf>. Acesso em: 26 ago. 2016. 
METCALF \& EDDY. Wastewater Engineering: Treatment and Reuse. 4 th. New York: McGraw-Hill, 2003.

NEOH, C.H.; NOOR, Z.Z.; MUTAMIM, N.S.A.; LIM, C.K. Green Technology in Wastewater Treatment Technologies: Integration of Membrane Bioreactor with Various Wastewater Treatment Systems. Chemical Engineering Journal, v.283, p.582-594. 2016. Disponível em: <http://dx.doi.org/10.1016/j.cej.2015.07.060> Acesso em: 14 set. 2016. doi: 10.1016/j.cej.2015.07.060

NUORTILA-JOKINEN, J.; MÄNTTÄRI, M.; HUUHILO, T.; KALLIOINEN, M.; NYSTRÖM, M. Water circuit closure with membrane technology in the pulp and paper industry. Water Science and Technology, v.50, n.3, p. 217-227, 2004. Disponível em: <https://www.ncbi.nlm.nih.gov/pubmed/15461416> Acesso em: 15 nov. 2016.

OLIVEIRA, C.R. Aplicação da ultrafiltração na reciclagem de água na indústria de celulose e papel. 2003. 89 p. Dissertação (Mestrado em Ciências Florestais) Universidade Federal de Viçosa, Viçosa, MG.

PACHECO, S.M.V.; SOARES, C.H.L. Biotransformation of a paper and cellulose factory effluent in the presence of laccase and horseradish peroxidase. Arq. Inst. Biol., v.78, n.4, p.581-585, 2011. Disponível em: <http://www.biologico.sp.gov.br/docs/arq/v78_4/villela.pdf>. Acesso em: 29 ago. 2016.

QUARTAROLI, L. Aplicação da flotação por ar dissolvido como pós-tratamento de efluente de lado ativado em uma indústria de papel e celulose. 2012. 95 p. Dissertação (Mestrado em Ciências Florestais). Universidade Estadual do CentroOeste, Irati, PR.

QUARTAROLI, L.; KURITZA, J. C.; CAVALLINI, G. S.; VIDAL, C. M. S.; SOUZA, J. B. Aplicação de polímeros aniônico e catiônico na flotação por ar dissolvido como forma de pós-tratamento de efluente de indústria de papel e celulose. Scientia Forestalis, v.2, n.101, p.57-67, mar. 2014. Disponível em: <http://www.ipef.br/publicacoes/scientia/leitura.asp?Article=06\&Number=101> Acesso em: 22 ago. 2016

QU, F.; LIANG, H.; WANG, Z.; WANG, H.; YU, H.; LI, G. Ultrafiltration membrane fouling by extracellular organic matters (EOM) of Microcystis aeruginosain stationary phase: Influences of interfacial characteristics of foulants and fouling mechanisms. Water Research, 46, p.1490-1500. 2012. Disponível em: http://dx.doi.org/10.1016/j.watres.2011.11.051> Acesso em: 29 ago. 2016 doi: 10.1016/j.watres.2011.11.051.

REYES, R.I.Q. Tratamento de efluente da produção de polpa celulósica kraft através de membranas. 2013. 64 p. Dissertação (Mestrado). Universidade Federal de Viçosa, Viçosa, MG.

SOUSA, C. A.; SILVA, C.M.; VIEIRA, N.M.; MOUNTEER, A.H.; AMARAL, M.S.; MARCOS ROGÉRIO TÓTOLA, M.R.; NUNES, W.G. Thermophilic treatment of paper 
machine white water in laboratory-scale membrane bioreactors, Desalination and Water Treatment, v.27, 1-3, p.1-7. 2011. Disponível em: <http://dx.doi.org/10.5004/dwt.2011.2023> Acesso em: 22 ago. 2016. doi: 10/5004/dwt.2011.2023 